\title{
Experimental and Numerical Study on Plastic Zone Variation ahead of Fatigue Crack Tip
}

\author{
Zhang Wei ${ }^{1, *}$, Zhou Daoqing ${ }^{1}$, and Cai Liang ${ }^{1}$ \\ ${ }^{1}$ School of Reliability and Systems Engineering, Beihang University, Beijing, 100191, China
}

\begin{abstract}
The plastic deformation ahead of crack tip is of great significance to analysis of the fatigue crack growth behaviour. Using the in-situ microscopy experiment technique, the variation of strain field in the vicinity of crack tip is investigated within load cycles at the small time scale. The contours of plastic zones are measured through the in-situ observation and digital image correlation (DIC). Finite element method (FEM) is also used to simulate the plasticity ahead of the crack tip. Furthermore, the numerical studies are extended to the single overload case to analyse the effect of large plastic zone on the subsequent crack growth. The evolution of residual stress is extracted by FEM simulation to explore the influence of plastic deformation before, during and after the single overload applied on the following crack propagation. Based on the FEM analysis, a model is proposed to approximate the size of the overload effect zone. Finally, some experimental data and numerical simulations are employed to validate this model.
\end{abstract}

\section{INTRODUCTION}

The plastic strain ahead of the crack tip has a significant effect on the fatigue crack propagation. The nonlinear loading interaction under the variable amplitude loading is usually considered to be determined by this local plastic deformation. Particularly, the retardation phenomenon caused by the overload is one typical and simple example. Furthermore, the constant amplitude loading with spike loads or overloads are very common in the engineering practice. Therefore, the studies on plastic zone ahead of fatigue crack tip under the overload condition has a great importance [1].

Irwin [2] make an assumption of small scale yielding ahead of crack tip, which makes it possible to analyse the metal material fatigue by using the linear elastic fracture mechanics theory. But this modification of small scale yielding is not used to explain the nonlinear load interaction at that time. Then, many studies are conducted to investigated the plasticity in the vicinity of crack tip [3][4], since it is considered as the key mechanism of the crack growth behaviour [5][6][7]. Dugdale and Barenblatt et al. also put forward a plastic zone estimation formula [8][9]. Irwin proposed a plastic zone size formulation, which could only calculate the plastic zone under the monotonic load [2]. Bannatine modified the Irwin method, which could estimate the plastic zone under cyclic loading. [10]. Suzuki determines the size of plastic zone by optical microscopy [11]. Paul simulates the size and shape of the monotonic and cyclic plastic zones by the numerical model [1].

In engineering practice, many structures are used under the variable amplitude loading and the variable loading induces the retardation which has influence on crack propagation. Therefore, many scholars studied the influence of retardation on crack growth. Sander et al. apply the overload testing on aluminum alloy compact tension specimens (CT) to obtain the relationship between the overload ratio and retardation effect [12]. Silva studies the fatigue crack growth retardation by overloads at negative stress ratios [13]. Kalnaus et al. investigate the fatigue crack propagation in AL6XN stainless steel to observe the obvious retardation phenomenon after the overload [14].

Plastic zone ahead of crack tip is usually used to analysis the nonlinear crack propagation under the overload condition. Willenborg model and wheeler model correlate the effective stress intensity factor range with the plastic zone size and predict the crack propagation after the overloading successfully [15][16]. Yuen and Taheri put forward a modified wheeler model, which can describe the retardation effect on crack propagation under the multiple overloads [17]. Additionally, the plasticity-induced closure is also a good alternative to deal with the crack growth under the overload. Zhang and Liu build up a relationship between the crack closure with the forward and reversed plastic zones [18]. Furthermore, Zhang and Jiang apply that closure model into the fatigue crack propagation prediction under variable amplitude loading [19][20].

However, there are some problems with the models and methods above. One is that the plastic zone' $\mathrm{s}$ model proposed by Irwin, this model is too simple to consider the influence from the actual sharp. And this models calculate plastic zone size under the monotonic loading condition only and ignore the effect of the previous residual stress. Secondly, the scope of plasticity influence is assumed the distance from the origin to the 
position where the new plastic zone reaches the boundary of the big plastic zone induced by the overload, which is much smaller than experimental observation [21][22].

In this paper, the in-situ experiment and FEM simulation are employed to investigate the variation and influence of the plastic zone in the vicinity of fatigue crack tip under the constant amplitude loading with an overload. The residual stress extracted by simulation is used to study the effect of the plasticity induced by the overload on the subsequent crack growth.

\section{Experiment and Simulation}

\subsection{Materials and specimen design}

The material used in this study is aluminium alloy 2024T3, which is a kind of hard aluminium with high strength and superior anti-fatigue property. The Young's modulus is 7.2GPa; the Poisson's ratio is 0.33 ; and the yield stress is $328 \mathrm{MPa}$.

The specimen is a thin plate with a single-edge crack, whose width $\mathrm{W}=12 \mathrm{~mm}$, length $\mathrm{L}=76 \mathrm{~mm}$, thickness $\mathrm{T}=1 \mathrm{~mm}$, and the initial crack $\mathrm{a}=1.45 \mathrm{~mm}$, as shown by Fig 1.

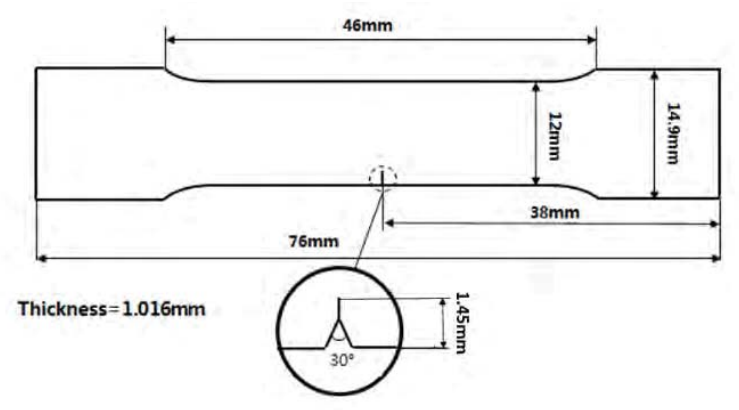

Fig. 1. The schematic illustration of specimen.

\subsection{Fatigue testing and DIC procedure}

The experimental system consists of a fatigue testing stage, an optical measuring microscope and a digital imaging processing system, as shown in Fig 2. Firstly, spray the matte paint onto the polished surface of the specimen with a very fine spray gun, and the speckles are required moderate size and evenly distributed around the crack tip, as shown in. Fig 3. After that, apply the cyclic loading with stress ratio $\mathrm{R}=0$ and maximum load $S_{\text {max }}=1.5 \mathrm{KN}$ onto the specimen by the fatigue testing machine. The optical measuring microscope is used to trace the local strain field ahead of the crack tip. During the measurement process, the in-situ images at different loading levels are taken and collected at a very high speed.

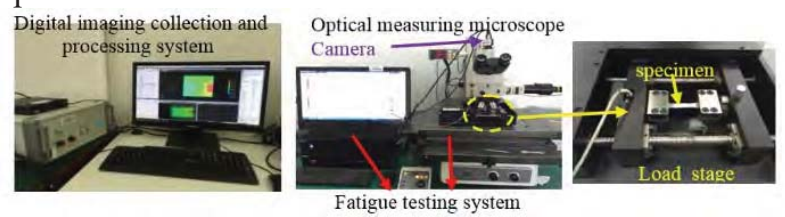

Fig. 2. The schematic illustration of experimental setup.

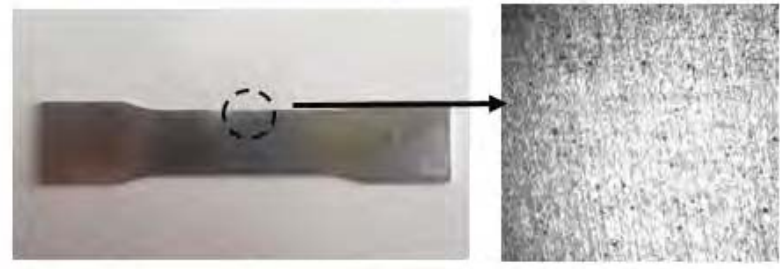

Fig. 3. The processed surface of the specimen.

The images are obtained, the variation of the strain ahead of crack tip can be analyzed through DIC (digital image correlation). The image feature points are recognized by DIC system, and the the strain field can be calculated based on the displacement field, as shown in Fig 4. Given the yield strain, the daimeter of the plastic zone can be measured. Repeat the above process at each loading level, variation of the plastic zone size can be traced.

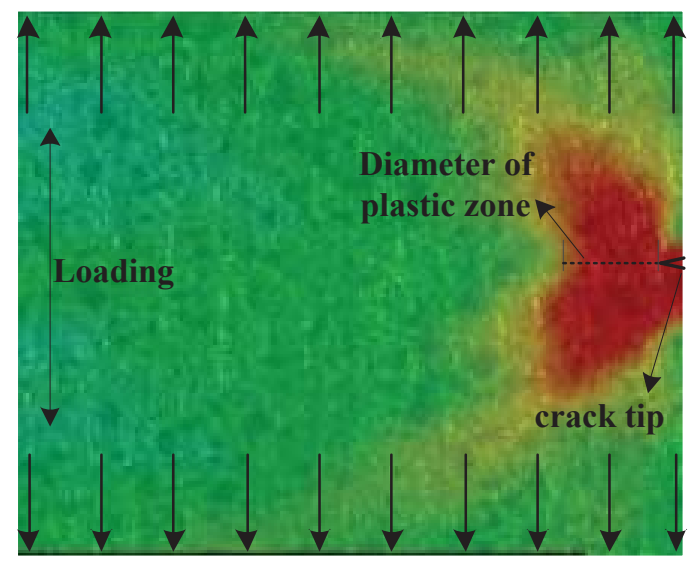

Fig.4. The relative strain field observed in the experiment

\subsection{FEM modelling}

Since the specimen is a very thin plate, it belongs to the plane stress problem. A 2D FEM model is built to simulate the variation of strain distribution in the vicinity of the crack tip. The specimen configuration, material properties, and loading/constraint conditions are kept equivalent to the real specimen in our experiment.

The constraints of the specimens are shown in Fig 5. Apply the constant loading with stress ratio $\mathrm{R}=0$ and maximum load $S_{\max }=1.5 \mathrm{KN}$. The variation of the stress-strain field and the diameter of plastic zone ahead of the crack tip are investigated. And the residual stress at the peak loads is computed to analyze the retardation effect region.

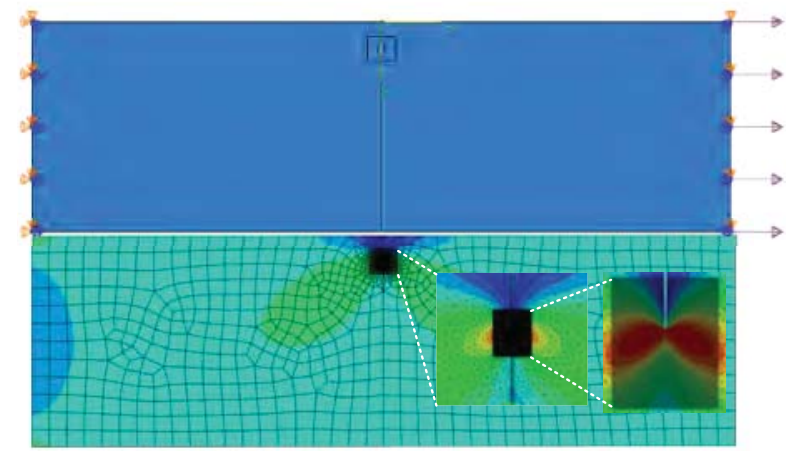

Fig. 5. The specimen's loading and restraint 


\subsection{Comparison between experiment and FEM model}

The stress-strain fields ahead of the crack tip are shown in Fig 6. The upper-plots are the experiment measurements, while the down-plots are the simulation results. The diameters of plastic zone are shown in Fig 7. The simulation matches the testing data well, which can verify the effective of this simulatin approach.

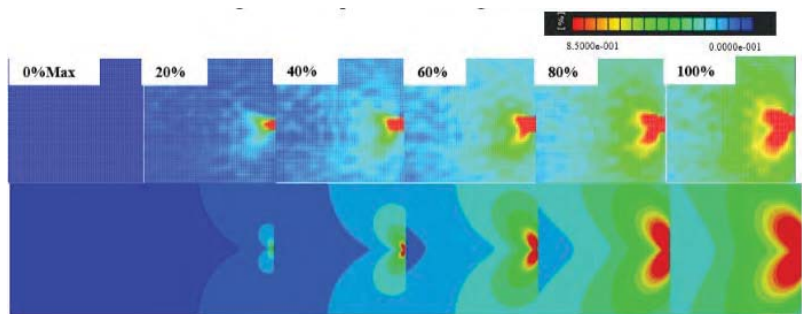

Fig. 6. The local strain evolution of experiment and simulation

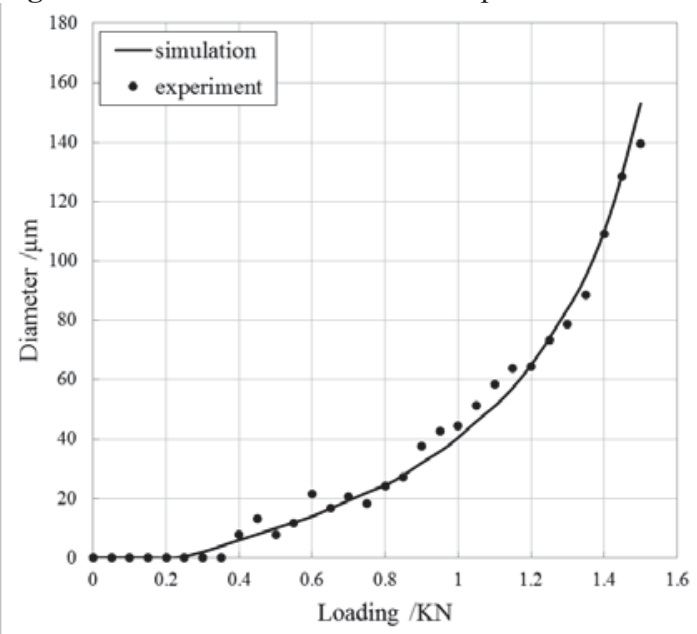

Fig. 7.The diameter of relative plastic zone.

\section{Modelling and validation}

\subsection{Residual stress analysis}

Through examining the influence of plasticity induced by the single overload on the subsequent crack growth, some experimental observations show that there are obvious differences between the effect region and its classical solution [23]. The classical models proposed by Irwin consider the shape of plastic zone is a circle, but the actual shape of plastic zone is observed as a butterfly shape by the expriment [24]. Therefore, the residual stress is used to investigate the effect region of plsticity.

The residual stress is obtained by the aforementioned simulation approach, which is used to analyze the variation of the plastic zone before, during and after a single overload. The schematic illustration of residual stress is shown in Fig 8.

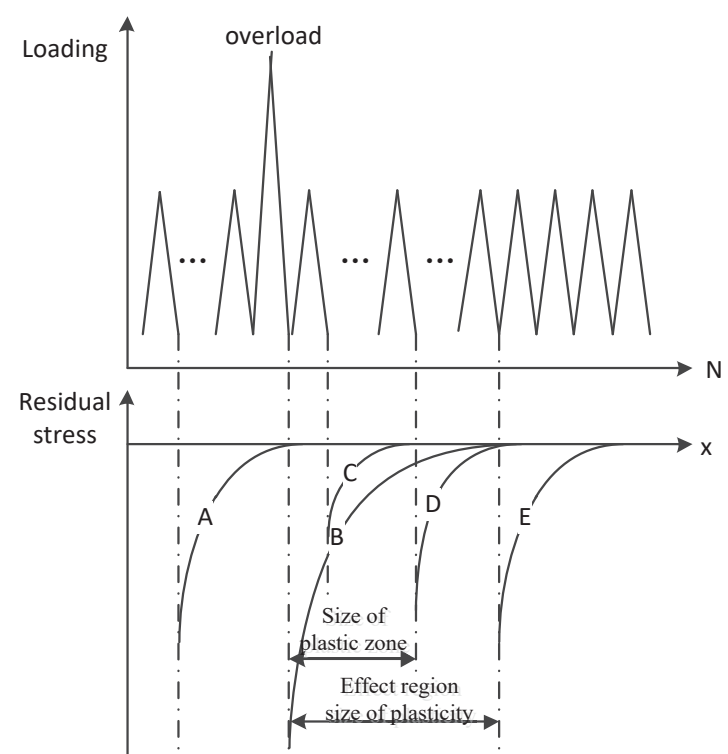

Fig. 8. The schematic illustration of residual stress.

Curve A represents the residual stress under the constant loading as the baseline. Once the overload is applied, residual stress changes greatly (the curve B). Afterwards, the residual stress is still affected by the overload (the curve C). Curve D is the residual stress at the boundary of the plastic zone calculated by the Irwin's model. The residual stress does not return back to the baseline. It indicates that this position is still in the effect region by the overload. Until the curve coincides with the curve $\mathrm{E}$, the loading sequence effect vanishes entirely. The distance from the curve B to the curve D is the plastic zone size calculated by Irwin's model. The distance from curve $\mathrm{B}$ to the curve $\mathrm{E}$ is the effect region size of the overload.

Noroozi et.al point out that the distance from the origin to the first intersection point of residual stress curve and the $\mathrm{X}$ axis is the size of plastic zone [23]. The distance is defined as the criterion to judge if the residual stress has returned back to the baseline. The residual stress of every cycle is computed and compared with the baseline. By this way, the effect region of large plastic zone can be found by the comparison of the residual stresses.

Take the load condition with $\mathrm{R}=0$ and $\mathrm{r}=1.2$ as an example, the procedure is shown in Fig 9. 


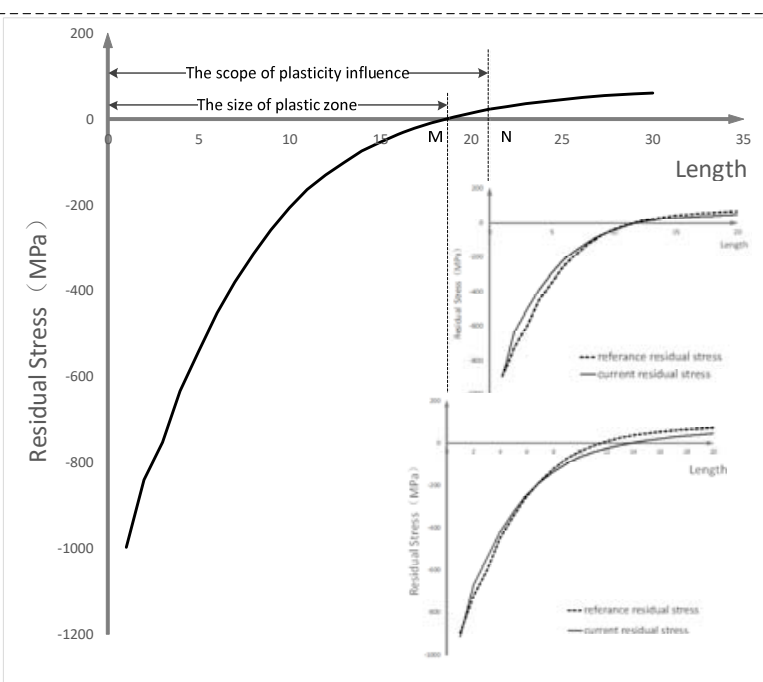

Fig. 9. Schematic illustration of the effect region $(\mathrm{r}=1.2 \& \mathrm{R}=0)$

Fig 9 shows the distribution of the residual stress along the crack direction. The point $\mathrm{M}$ is the boundary of plastic zone calculated by Irwin's model, where the residual stress has not returned back to the baseline. The residual stress at point $\mathrm{N}$ basically reverts to the original level. So the point $\mathrm{N}$ is considered as the boundary, at which the overload has no influence on the subsequent crack. The distance from the origin to the point $\mathrm{M}$ is the size of plastic zone. And the distance from the origin to the point $\mathrm{N}$ is the effect region size.

\subsection{Modelling}

For normalization, the ratio between the effect region size of the plastic zone and its classical solution is defined as $\varphi$, which is a function of overload ratio $r$ and the stress ratio $R$. This equation is developed as following.

First of all, the overload ratio remains the same, the stress ratio is set as $0,0.3,0.5$ and 0.7 . The ratios between the effect region and its classical solution are calculated and fitted by the equation $f(R)$. The fitting diagram under the loading of the stress ratios $0,0.3,0.5$ and 0.7 is depicted below in Fig. 10-13.

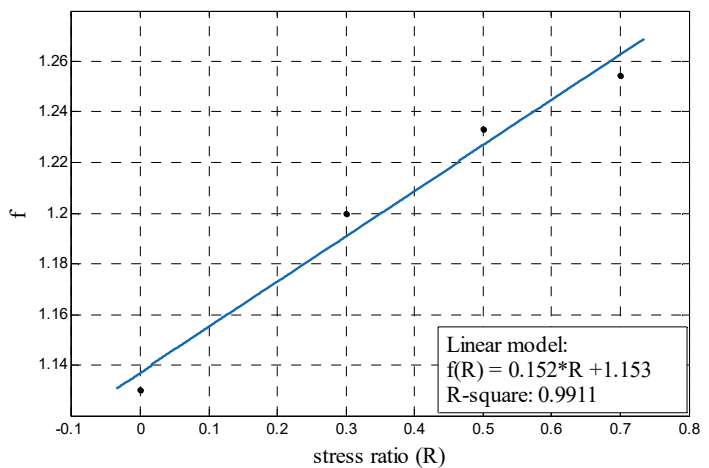

Fig. 10. The fitting diagram $(\mathrm{R}=0 \& \mathrm{r}=1.2)$

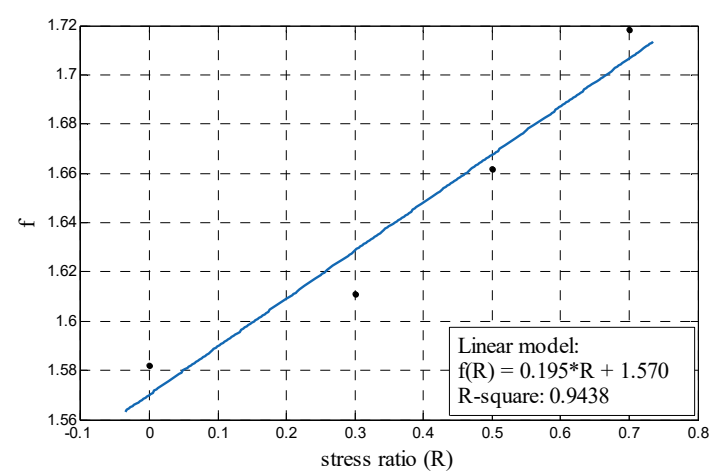

Fig. 11. The fitting diagram $(\mathrm{R}=0 \& \mathrm{r}=1.5)$

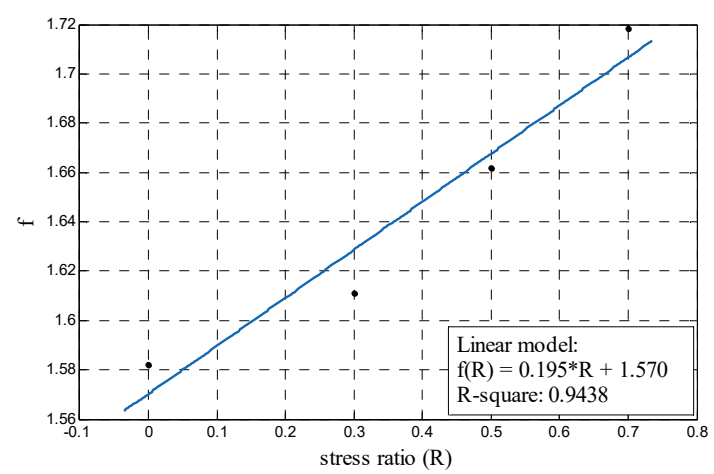

Fig. 12. The fitting diagram $(\mathrm{R}=0 \& \mathrm{r}=1.8)$

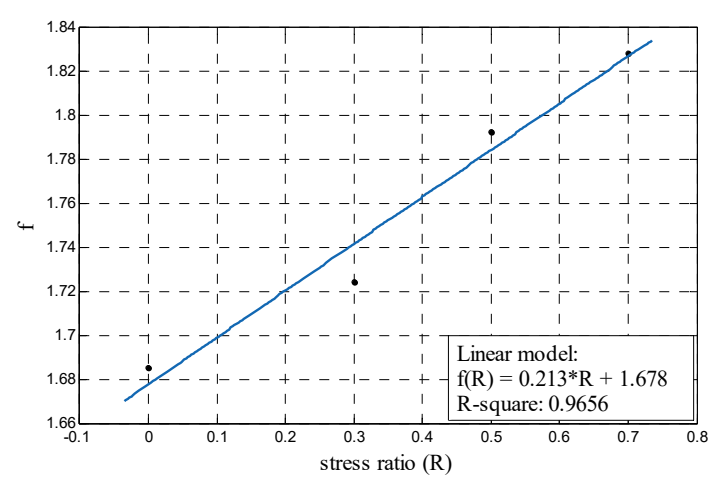

Fig. 13. The fitting diagram $(\mathrm{R}=0 \& \mathrm{r}=2.0)$

From the Fig 10-13, the relationship between them is linear. The fitting equation can be expressed as $f(R)=A * R+B$, where the values of $A$ and $B$ are related to the stress ratio and overload ratio.

Thus, $\mathrm{A}$ and $\mathrm{B}$ can be determined by equations of overload ratio. The value of $\mathrm{A}$ and $\mathrm{B}$ under different overload ratios are expressed as the fitting equation $\mathrm{p}(\mathrm{r})$ and $\mathrm{q}(\mathrm{r})$. And the fitting diagrams are demonstrated in Fig.14 and Fig.15.

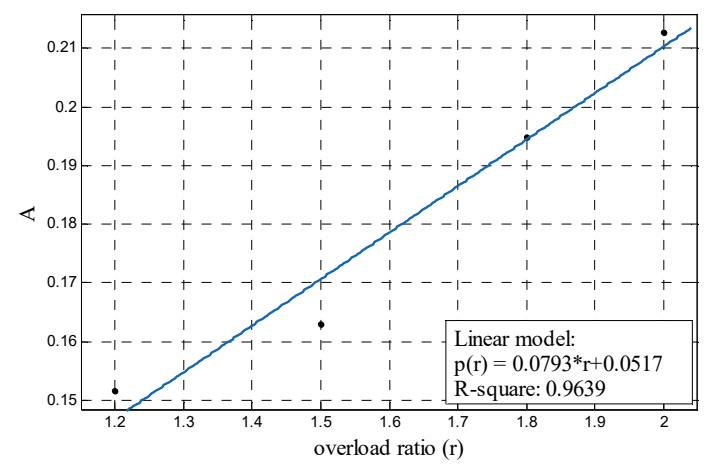


Fig. 14. The fitting diagram of parameter A

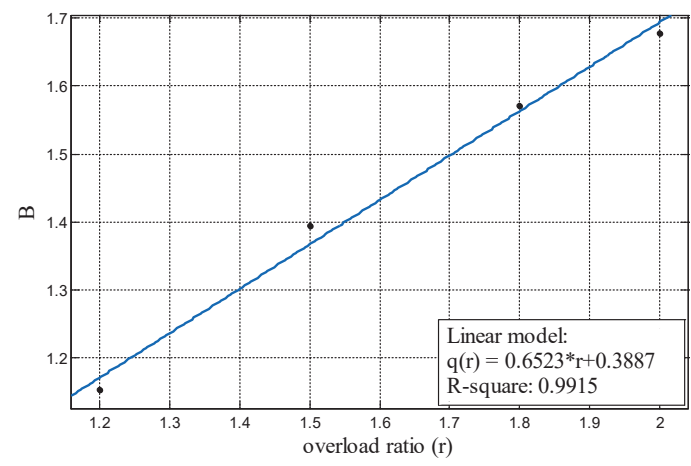

Fig. 15. The fitting diagram of parameter $B$

Substitute this result into the equation $\varphi(R, r)$, the equation can be rewritten as:

$$
\begin{array}{rl}
\varphi(R, r)=p(r) * & R+q(r) \\
& =(0.0793 * r+0.0517) * R \\
& +(0.6523 * r+0.3887)
\end{array}
$$

This model can calculate the effect region size under tension-tension loading condition (the range of overload ratio is $1 \leqslant \mathrm{r} \leqslant 2.3)$ [25]. The value of $\varphi(R, r)$ is indirect proportion to the stress ratio and the overload ratio respectively. When the overload ratio is 1, this formulation describes the plastic effects under constant amplitude loading, which is apparently larger than the classical solution. It further manifests that the plastic zone shape has a noteworthy influence on the plasticity effect area. In addition, the overload ratio is a more sensitive factor of $\varphi(R, r)$, comparing with the stress ratio. Therefore, the plastic effect area under different overload ratio is investigated below.

\subsection{Validation}

\section{Experiment}

The experimental data of aluminum alloy D16 are used to validate the proposed model [21]. The initial center crack length is $20 \mathrm{~mm}$. The mechanical properties of D16 in longitudinal (LT) orientation are as follows: $\sigma_{\mathrm{y}}$ $=345 \mathrm{MPa}, \sigma_{\text {UTS }}=483 \mathrm{MPa}$. The crack increment $\Delta \mathrm{a}$ under the overload effects is shown in Fig 16.

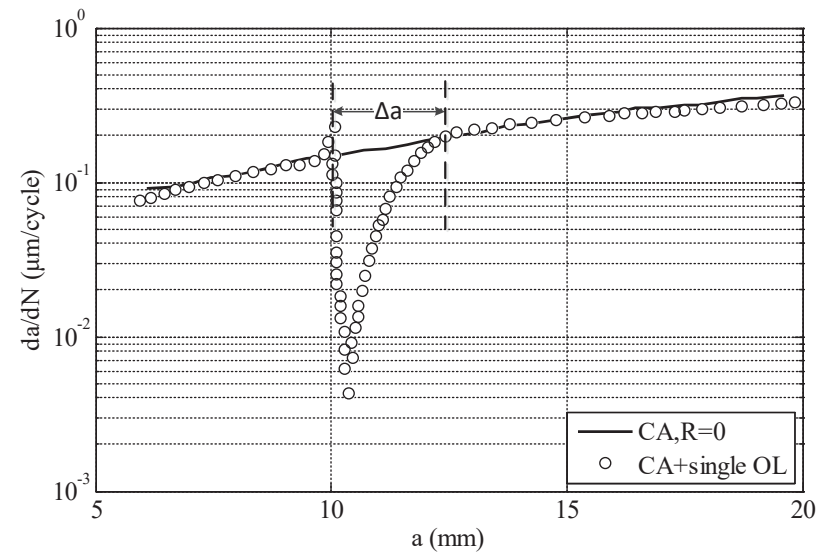

Fig. 16. The retardation after a single overload in D16
The crack length a is measured in Fig 15, and the details of the testing can be found in the paper [21]. The maximum stress intensity factor $K_{\max }$ is $22.89 \mathrm{MPa} *$ $(m)^{\frac{1}{2}}$.

According to the Irwin's model [2], the plastic zone ahead of crack tip can be calculated:

$$
\mathrm{L}=\left(\frac{K_{\max }}{\sigma_{y}}\right)^{2} * \frac{1}{\pi}=14.86 * 10^{-4} \mathrm{~m}
$$
16:

The size of effect region is measured from the Fig

$$
\mathrm{d}=2.5 * 10^{-3} \mathrm{~m}
$$

Then, the ratio between the effect region and its classical solution is obtained:

$$
\rho=\frac{\mathrm{d}}{L}=1.6822
$$

The ratio by the proposed model $\varphi(R, r)$ is:

$$
\varphi(0,2)=1.6933
$$

It is noted that the proposed model can give reasonable predictions under the constant loading with overload.

\section{Simulation}

In addition, a simulation is conducted to further validate the aforementioned approach. The plastic effect region $\alpha$ is calculated by the proposed formulation of $\varphi(R, r)$. $\beta$ is defined as the distance from the origin to the position where the residual stress return back to the baseline (the constant loading condition). The values of $\alpha$ and $\beta$ are compared under different overload ratio cases.

This simulation example is considered as a single edge crack. Hence, the maximum stress intensity factor, $K_{\text {max }}$, is expressed as:

$$
\begin{aligned}
K_{\max }=\sigma \sqrt{\pi a}[1.12 & -0.23 *\left(\frac{a}{b}\right)+10.6 *\left(\frac{a}{b}\right)^{2} \\
- & \left.21.7 *\left(\frac{a}{b}\right)^{3}+30.4 *\left(\frac{a}{b}\right)^{4}\right]
\end{aligned}
$$

The size of plastic zone is calculated by Irwin's model [2], which can be written as:

$$
\mathrm{L}=\left(\frac{K_{\max }}{\sigma_{y}}\right)^{2} * \frac{1}{\pi}
$$

The diameter of effect region $\alpha$ is calculated as:

$$
\alpha=\varphi(0.5,1.2) * L
$$

Moreover, $\beta$ is measured from the figures directly.

So the comparison between the distances $\alpha$ and $\beta$ is obtained, and the relative error $\delta$ is expressed as:

$$
\delta=\left|\frac{\alpha-\beta}{\alpha}\right|
$$

The validations are shown in Fig 17 and Fig 18. 


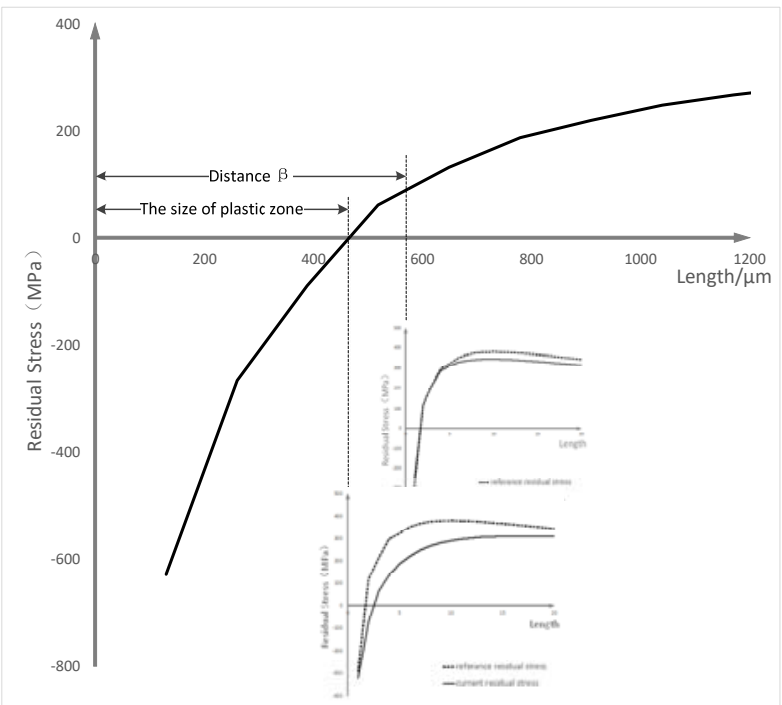

Fig. 17. Schematic illustration of effect region $(r=1.2 \& R=0.5)$

Under the loading of the $\mathrm{R}=0.5$ and $\mathrm{r}=1.2$, the parameters are calculated as following:

$$
\begin{gathered}
\alpha=\varphi(0.5,1.2) * L=548.568 \mu \mathrm{m} \\
\beta=580 \mu \mathrm{m} \\
\delta=\left|\frac{\alpha-\beta}{\alpha}\right|=5.8 \%
\end{gathered}
$$

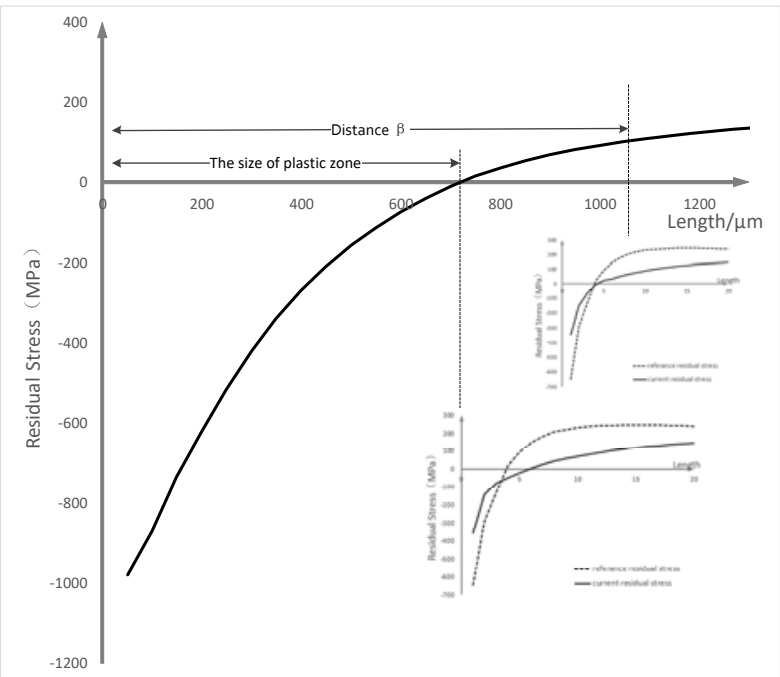

Fig. 18. Schematic illustration of effect region $(r=1.5 \& R=0.3)$

Under the loading condition of $\mathrm{R}=0.3$ and $\mathrm{r}=1.5$, the parameters are calculated as following:

$$
\begin{gathered}
\alpha=\varphi(0.3,1.5) * L=976.57 .57 \mu \mathrm{m} \\
\beta=1040 \mu \mathrm{m} \\
\delta=\left|\frac{\alpha-\beta}{\alpha}\right|=6.5 \%
\end{gathered}
$$

The process above proves that the proposed model matches the simulation well.

\section{Conclusion}

The in-situ optical microscopy testing is performed to measure the variation of strain distribution ahead of the crack tip under the constant amplitude loading. A FE model is established to simulate this process, and a very good agreement is received. Then, the FE model is employed to analyse the plasticity and residual stress caused by the overload around the crack tip. A new calculation model is proposed to estimate the retardation effect region under the constant amplitude loading with a single overload. Some conclusions can be drawn:

- The effect region size is larger than its classical solution.

- This model can calculate the size of retardation effect region under the constant loading and overload more accurately, which is validated by experiment and simulation.

In the future work, the experimental and numerical investigation will be extended to more complex loading case.

The research is financially supported by the National Natural Science Foundation of China (Grant No. 51405009).

\section{References}

1. S.K. Paul, E.F.M. 159, 90-97(2016)

2. H. Tada, P.C. Pairs, G.R. Irwin, D.R.C., H.P. (1973)

3. W.Y. Maeng, M.H. Kim, J.N.M, 282, 32-39(2000)

4. Y.Xiong, X.X. Hu, J. Katsuta, et al.,I.J.F, 30, 6773(2008)

5. D.L. Davidson, J. Lankford, T. Yokobori, et al., I.J.F, 12, 579-585(1976)

6. G. Xin, H. Wang, X. Kang, et al., E.J.M.A.S., 29, 739-745(2010)

7. R.A. Sousa, F.P. Figueiredo, F.F.E.M.S, (2017)

8. D.S. Dugdale, J.M.P.S, 8, 100-104(1960)

9. G.I. Barenblatt, A.A.M, 7, 55-129(1962)

10. J. Bannantine, P.H., 70, 219-225(1990)

11. H. Suzuki, A.J. Mcevily, M.M.T.A, 10, 475481(1979)

12. M. Sander, H.A. Richard, I.J.F, 25, 999-1005(2003)

13. F.S. Silva, I.J.F., 29, 1757-1771(2007)

14. S. Kalnaus, F. Fan, A.K. Vasudervan, et al. E.F.M., 75, 2002-2019(2008)

15. J. Willenborg, R.M. Engle, H.A. Wood, T.R.A.(1979)

16. O.E. Wheeler, J.B.E., 94, 181-186(1972)

17. B.K.C. Yuen, F.Taheri, I.J.F., 28, 1803-1819(2006)

18. W. Zhang, Y. Liu, I.J.F., 43, 188-198(2012)

19. S. Jiang, W. Zhang, Z.L. Wang, J.V. 17, 36503661(2015)

20. S. Jiang, W. Zhang, X. Li, et al., M.P.E, 4, 19(2014)

21. J. Schijve, M. Skorupa, A. Skorupa, I.J.F., 26, 115(2004)

22. T. Jian. A. Ping, et al., S.C.T.S., 55, 673-683(2012)

23. A.H. Noroozi, G. Glinka, S. Lambert, E.F.M., 75, 188-206(2008)

24. M. Besel, E. Breitbarth, I.J.F.,93, 92-108(2016)

25. J.P. Gallagher, A.G.D.Y.Z.M, (1974) 